\title{
Dosage rapide du citrate dans l'ultrafiltrat de lait par complexation cuivrique
}

\author{
par \\ A. PIERRE et G. BRULE
}

\section{Rés u m é}

Les propriétés complexantes du citrate vis-à-vis du cuivre étaient utilisées pour la mise au point d'une méthode de détermination de la teneur en citrate du lait et des produits laitiers. La détection du complexe citrate- $\mathrm{Cu}$ était effectuée par mesure de l'absorption à 280 $\mathrm{nm}$. Les conditions optimales de $\mathrm{pH}$, de température et de concentration en $\mathrm{Cu}$ et en citrate étaient déterminées à partir de leurs effets respectifs sur l'absorption. L'application de ce dosage aux produits laitiers était étudiée ainsi que les interférences possibles liées aux constituants propres du lait (phosphate, lactate, protéine). La méthode proposée permet de doser le citrate soluble dans l'ultrafiltrat de lait avec une précision équivalente à celle des autres méthodes existantes.

Mots clés :

Dosage du citrate - Cuivre - Lait.

\section{S u m m a ry}

\section{CITRATE DETERMINATION IN MILK ULTRAFILTRATE} WITH COPPER-COMPLEX METHOD

A method for citrate determination was studied; it was based up on complexing properties of citrate for $\mathrm{Cu}^{++}$ions. The detection was made by absorptiometry at $280 \mathrm{~nm}$. Optimal conditions for $\mathrm{pH}$, temperature and relative concentrations of $\mathrm{Cu}$ and of citrate through their effects on absorption were investigated. Adaptation of the

Institut National de la Recherche Agronomique, Laboratoire de Recherches de Technologie Laitière, 65, rue de Saint-Brieuc - 35042 Rennes cedex. 
method to milk and dairy products was studied and possible interferences of other milk components on the reaction were reviewed. The Cu-complex method allows a good recovery and determination of diffusible citrate in milk. Sensibility and accuracy were found similar to reference methods, but the proposed method is easier and faster.

Key words:

Citrate determination - Copper - Milk.

\section{INTRODUCTION}

L'étude des équilibres minéraux du lait nécessite souvent la détermination du taux de citrate. Il existe, en effet, une corrélation entre la concentration de cet anion et celle du calcium soluble (Holt et Muir, 1979). La méthode décrite par Marier et Boulet (1958) puis modifiée par White et Davies (1963) est habituellement utilisée pour le dosage du citrate du lait ou des produits laitiers (Pyne et Mc Gann, 1960 ; Holt et Muir, 1979). Cette méthode est précise et spécifique mais nécessite une incubation et l'utilisation de réactifs anhydres et corrosifs. Deux autres méthodes ont été proposées récemment : l'une par voie enzymatique (Mutzelburg, 1979) et l'autre par H.P.L.C. (Marsili et al., (1981). Elles sont fiables et très spécifiques mais exigent l'emploi de réactifs très onéreux. Nous nous sommes proposés d'étudier une méthode rapide et facile à mettre en œuvre. Elle est basée sur la très grande affinité du citrate vis-à-vis du cuivre qui conduit à la formation d'un complexe possédant un coefficient d'extinction moléculaire très élevé.

\section{MATERIEL ET METHODES}

Les solutions de citrate ont été préparées à partir de citrate trisodique et les ions $\mathrm{Cu}^{++}$ajoutés sous forme d'une solution de chlorure de cuivre, $\mathrm{CuCl}_{2}$. Le complexe $\mathrm{Cu}$-citrate a été étudié dans les solutions contenant de 0.05 à $0.5 \mathrm{mM} / 1$ de citrate et de 0.1 à $4 \mathrm{mM} / 1$ de $\mathrm{Cu}^{++}$. La formation du complexe a été détectée grâce à ses propriétés d'absorption dans la région UV du spectre. L'absorption était lue à $280 \mathrm{~nm}$ à l'aide d'un spectrophotomètre Beckman ACTA MVI (Beckman Instruments France, 52/54, chemin des Bourdons - 93220 Gagny [France]). Les ultrafiltrats ont été obtenus à partir de laits crus ultrafiltrés sur des membranes coniques Centriflo CF 25 (Amicon S.A.R.L., 63/65, place de la Réunion, Paris [France]) et centrifugés sur une centrifugeuse MSE (2000 g - $\left.90 \mathrm{~min}-20^{\circ} \mathrm{C}\right)$. Les protéines ont été précipitées avec des solutions d'acide trichloracétique (TCA) à $15 \%$, la concentration finale en TCA étant de $12 \%$. 


\section{RESULTATS ET DISCUSSION}

\section{Facteurs affectant l'absorption du complexe Cu-citrate}

$p H$ : Les effets du $\mathrm{pH}$ sur l'absorption du complexe ont été étudiés à l'aide d'une solution de citrate de sodium $(0.5 \mathrm{mM} / \mathrm{l})$ à laquelle on a ajouté du chlorure de cuivre, $\mathrm{CuCl}_{2}(2 \mathrm{mM} / \mathrm{l})$. Le $\mathrm{pH}$ était progressivement modifié par addition de soude $0.25 \mathrm{M}$ et l'absorption notée dans la gamme de $\mathrm{pH} 4.0$ à $\mathrm{pH}$ 6.4. Dans un autre essai, on ajoutait $0.5 \mathrm{mM} / \mathrm{l}$ de phosphate de sodium à la solution de citrate pour simuler la composition de la phase aqueuse du lait. Les résultats sont présentés dans la figure 1. L'absorption du complexe Cu-citrate augmentait à partir de $\mathrm{pH} 4$ jusqu'au $\mathrm{pH} 6$; à des $\mathrm{pH}$ plus élevés, une précipitation de $(\mathrm{OH})_{2} \mathrm{Cu}$ se produisait. Quand on ajoutait du phosphate de sodium au citrate, l'absorption n'était pas modifiée pour

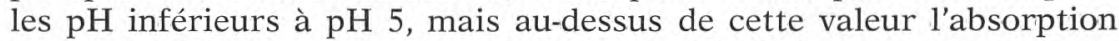
augmentait fortement sans précipitation. Ainsi, la détermination du

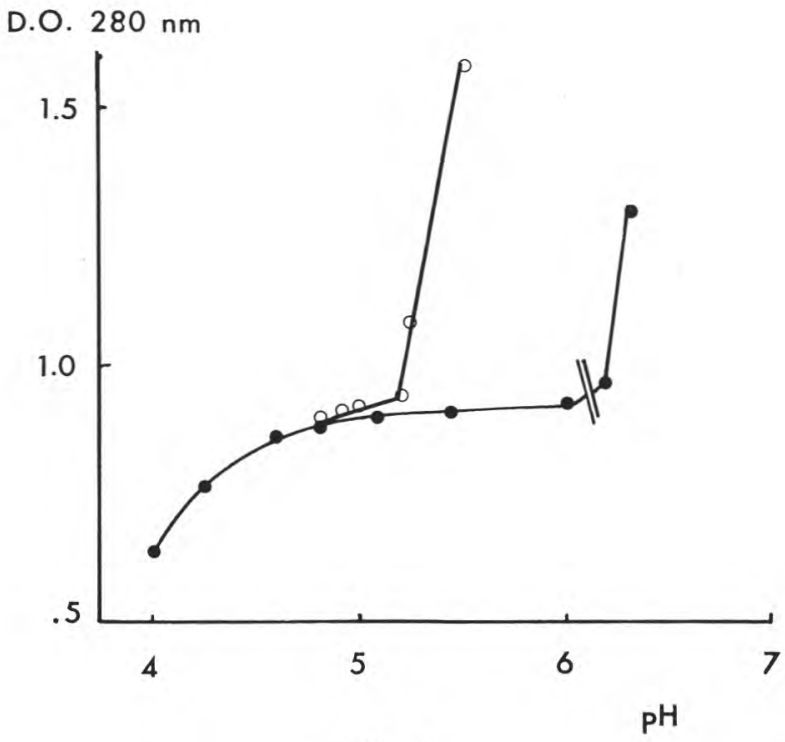

Fig. 1

Absorption du complexe citrate-cuivre en fonction du $\mathrm{pH}$; $(\bullet)$, citrate $0,5 \mathrm{mM} / 1$ et rapport cuivre/citrate $=4$; (0), citrate $0,5 \mathrm{mM} / 1+$ phosphate $0,5 \mathrm{mM} / 1$ et rapport cuivre/citrate + phosphate $=2$.

Absorption of the Cu-citrate complex as a function of $p H$; $0.5 \mathrm{mM}$ citrate, $\mathrm{Cu} /$ citrate ratio $=4(\bullet) ; 0.5 \mathrm{mM}$ citrate + $0.5 \mathrm{mM}$ phosphate, $\mathrm{Cu} /$ citrate + phosphate ratio $=2(0)$. 
citrate en présence de $\mathrm{PO}_{4} \mathrm{H}^{-} 2$ ne peut se faire qu'à $\mathrm{pH}$ compris entre 4 et 5. Dans les essais suivants on a donc choisi une valeur de $\mathrm{pH}$ de 4.9-5.0 ; l'ajustement à cette valeur a été fait par addition d'un tampon acétate $0.02 \mathrm{M}$ à $\mathrm{pH} 4.9$.

Concentration relative en $\mathrm{Cu}^{++}$et citrate : La figure 2 montre les variations de l'absorption mesurée dans deux expériences réalisées avec deux concentrations de citrate $(0.05 \mathrm{mM} / 1$ et $0.5 \mathrm{mM} / 1)$ et avec des quantités de $\mathrm{CuCl}_{2}$ croissantes. En raison de l'augmentation de l'absorption spécifique du tampon avec la teneur en cuivre, la valeur de l'absorption du blanc a été déduite des valeurs présentées. Dans les solutions de citrate, quand la teneur en $\mathrm{Cu}$ est faible, l'absorption du citrate dépend de la quantité de $\mathrm{Cu}$. Pour des concentrations en cuivre égales ou supérieures à 2 ou $3 \mathrm{mM}$, l'absorption spécifique est indépendante de la quantité de $\mathrm{Cu}$. Une quantité de $\mathrm{Cu}$ de $2 \mathrm{mM} / \mathrm{l}$ a été retenue pour les essais ultérieurs correspondant à un rapport molaire $\mathrm{Cu} /$ citrate compris entre 4 et 40 quand la concentration en citrate varie de 0.5 à $0.05 \mathrm{mM} / 1$.

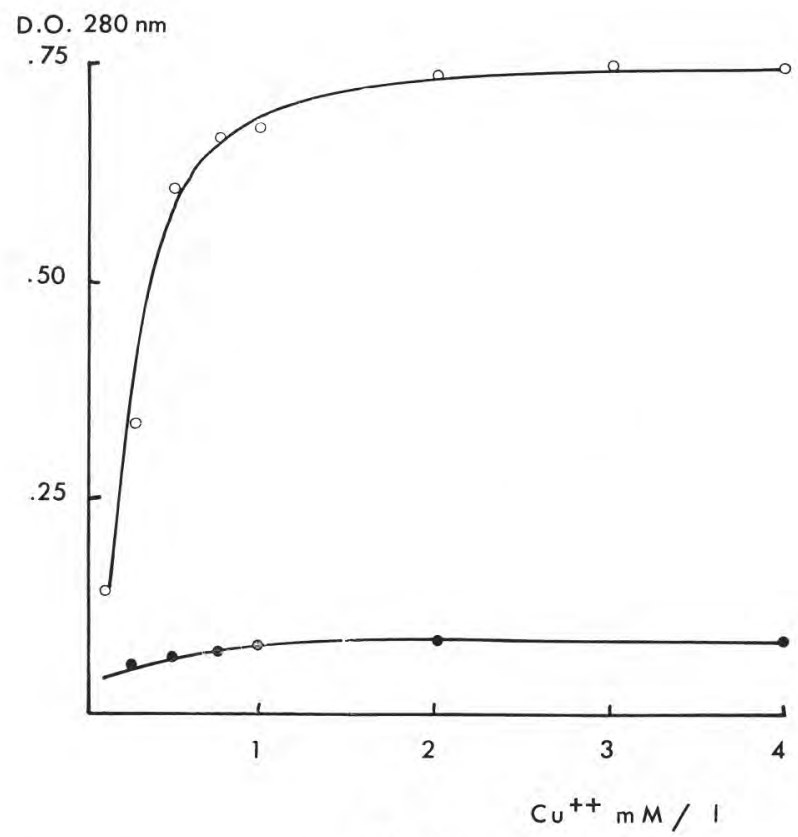

Fig. 2

Effet de la teneur en cuivre sur l'absorption du complexe citrate-cuivre pour deux concentrations de citrate, $(\bullet, 0,05$ $\mathrm{mM} / 1 ; 0,0,5 \mathrm{mM} / \mathrm{l})$.

Effect of Cu content on absorptivity of Cu-citrate complex for 2 citrate levels $(\bullet, 0.05 \mathrm{mM} ; 0,0.5 \mathrm{mM})$. 
Température : L'effet de la température sur l'absorption du complexe a été étudié entre $10^{\circ} \mathrm{C}$ et $30^{\circ} \mathrm{C}$. L'absorption du blanc augmentait de 0.0033 D.O. par degré, alors que l'absorption du complexe Cu-citrate n'était pas affectée par la température.

\section{Forme finale du dosage}

\section{Réactifs :}

- solution de $\mathrm{CuCl}_{2} 0.02 \mathrm{M}$;

- tampon acétate $0.02 \mathrm{M}, \mathrm{pH} 4.9$ : (40 ml d'acide acétique $0.2 \mathrm{M}$ et $60 \mathrm{ml}$ d'acétate de sodium $0.2 \mathrm{M}$ pour $1000 \mathrm{ml}$ ) ;

- solution étalon de citrate trisodique $0.01 \mathrm{M}$.

Méthode : l'échantillon contenant 0.25 à $2.5 \mu \mathrm{M}$ de citrate pour un volume maximal de $1 \mathrm{ml}$ est placé dans un tube gradué avec $0.5 \mathrm{ml}$ de la solution de $\mathrm{CuCl}_{2}$ et $3.5 \mathrm{ml}$ de tampon. Le volume total est

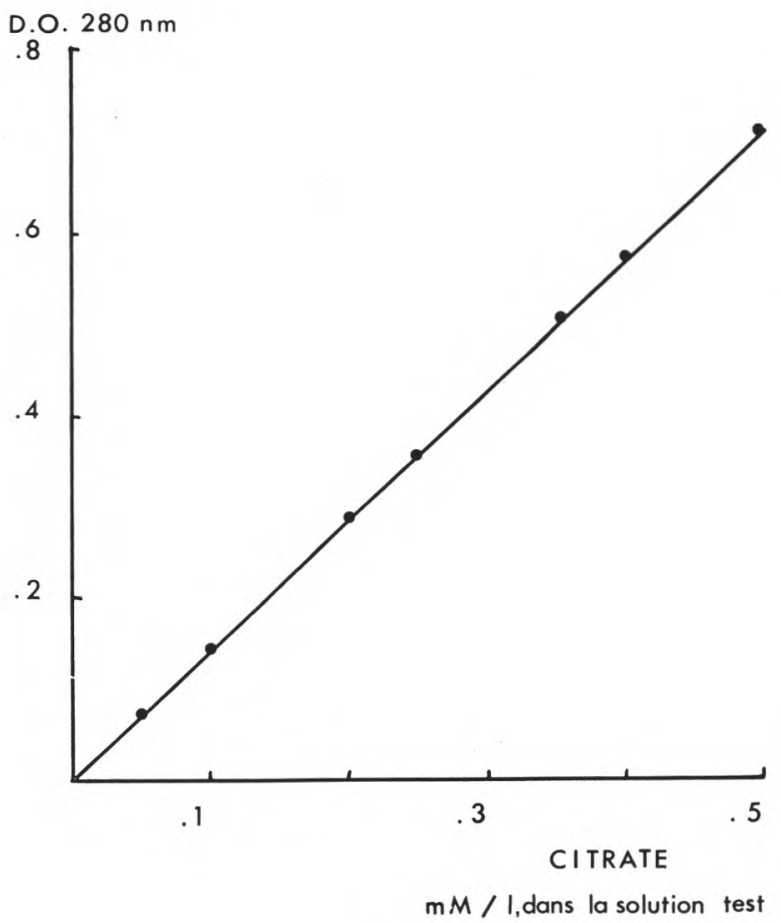

Fig. 3

Courbe d'étalonnage pour le dosage du citrate.

Standard curve for citrate determination. 
ajusté à $5 \mathrm{ml}$ par addition d'eau distillée, puis le contenu du tube agité. Des blancs sont préparés, l'un avec les réactifs seuls, l'autre avec l'échantillon et tous les réactifs excepté la solution de $\mathrm{Cu}$.

L'absorption à $280 \mathrm{~nm}$ peut être lue immédiatement. Une courbe étalon est présentée sur la figure 3.

\section{Application au lait et aux produits laitiers}

La validité du dosage sur le lait est soumise à plusieurs conditions. D'une part, il faut connaître la réactivité des ions $\mathrm{Cu}^{++}$vis-à-vis des autres solutés du lait, d'autre part, la matière grasse et la caséine doivent être enlevées à cause de leurs propriétés de diffraction de la lumière.

L'effet de certains acides présents dans le lait a été déterminé sur une solution de citrate à $0.25 \mathrm{mM} / 1$. Le coefficient d'extinction moléculaire du citrate dans les conditions de l'essai est de 1416 $\left(\mathrm{cm}^{-1}, \mathrm{M}^{-1}\right)$. L'addition de phosphate à une concentration de 0.05 à $0.9 \mathrm{mM} / 1$ dans la solution test augmente l'absorption. A partir de nos résultats, le coefficient d'extinction moléculaire du phosphate a été estimé à 48 . Pour des teneurs en phosphate supérieures à $0.9 \mathrm{mM} / 1$, il se produit une précipitation. L'addition de lactate (sel de lithium) aux concentrations de 0.5 à $2.4 \mathrm{mM} / 1$ dans la solution test augmentait aussi l'absorption (coefficient d'extinction moléculaire calculé de 51).

Les protéines ont une forte réactivité vis-à-vis du $\mathrm{Cu}$. Quand des protéines sont présentes dans la solution test pour la détermination du citrate, une augmentation de densité optique est observée, due en partie aux acides aminés aromatiques constitutifs des protéines, mais également à la formation d'un complexe protéines-cuivre. Des essais pour déterminer le coefficient d'extinction moléculaire des protéines ont montré que, dans les conditions du dosage, l'augmentation de densité optique n'était pas proportionnelle à la concentration en protéines. Il faut donc les éliminer de la solution avant la détermination du citrate.

La séparation peut être réalisée par une précipitation dans le TCA à $12 \%$; le filtrat étant très acide, il doit être neutralisé avant d'analyser sa teneur en citrate. En raison de cet inconvénient, nous avons finalement choisi de séparer les protéines par une ultrafiltration (tab. 1).

Par ailleurs, des additions de quantités connues de citrate ont été faites à des ultrafiltrats et l'analyse des échantillons a permis de retrouver les quantités ajoutées avec une précision de $3 \%$. La comparaison avec la méthode de White (1963) a été faite en analysant 32 échantillons d'ultrafiltrat de laits individuels. Les résultats (tab. 2) montrent une bonne corrélation entre les valeurs individuelles obtenues par les deux méthodes $(r=0.960)$. La répétabilité de la méthode d'un jour à l'autre a aussi été vérifiée en analysant le même échan- 
tillon d'ultrafiltrat 16 fois ; un coefficient de variation de $2.9 \%$ a été obtenu (tab. 3) très similaire à celui de la méthode de White et. Davies (1963).

\section{TABLEAU 1 - TABLE 1}

Comparaison des teneurs en citrate trouvées dans le lait ou le rétentat en fonction du procédé utilisé pour éliminer les protéines

(précipitation ou ultrafiltration)

Comparison of citrate levels in milks and ultrafiltrated milks (retentates) obtained after removal of proteins by 2 different procedures

\begin{tabular}{c|c|c|cc}
\hline \multirow{2}{*}{ Echantillon } & Produit & $\begin{array}{c}\text { Teneurs en } \\
\text { protéines } \\
(\mathrm{g} / \mathrm{kg})\end{array}$ & $\begin{array}{c}\text { Teneur en citrate (mM/l) dans le filtrat } \\
\text { après traitement par } \\
\text { ultrafiltration }\end{array}$ \\
\cline { 2 - 4 } 1 & TCA 12\% & 31,9 & 12,61 & 12,07 \\
2 & rétentat & 52,2 & 13,00 & 12,35 \\
3 & "ait & 86,5 & 13,77 & 12,61 \\
4 & lait & 35,4 & 7,57 & 7,97 \\
5 & rétentat & 66,4 & 8,82 & 8,48 \\
6 & " & 109,8 & 8,82 & 9,07 \\
\hline
\end{tabular}

TABLEAU 2 - TABLE 2

Comparaison des teneurs en citrate obtenues par deux méthodes dans des ultrafiltrats de laits individuels

Comparison of citrate levels determined by 2 methods in ultrafiltrates from individual milks

\begin{tabular}{l|c|c}
\hline & $\begin{array}{c}\text { Méthode } \\
\text { de } \\
\text { White et Davies }\end{array}$ & $\begin{array}{c}\text { Méthode de } \\
\text { complexation } \\
\text { au cuivre }\end{array}$ \\
\cline { 2 - 3 } Nombre d'échantillons & 32 & 32 \\
Valeur moyenne (mM/l) & 7,49 & 7,71 \\
Valeur minimum & 4,95 & 5,10 \\
Valeur maximum & 11,00 & 10,86 \\
Coefficient de corrélation & & 0,960 \\
\hline
\end{tabular}




\section{TABLEAU $3-T A B L E 3$}

Reproductibilité des résultats dans la détermination de la teneur en citrate d'un ultrafiltrat

Reproducibility of 2 methods in the analysis of citrate in a simple sample of ultrafiltrate

\begin{tabular}{l|c|c}
\hline & $\begin{array}{c}\text { Méthode } \\
\text { de } \\
\text { White et Davies }\end{array}$ & $\begin{array}{c}\text { Méthode de } \\
\text { complexation } \\
\text { par le cuivre }\end{array}$ \\
\hline Valeur moyenne (mM/l) & 7,3 & 6,8 \\
Ecart-type & .19 & .20 \\
Nombre d'analyses & 16 & 16 \\
Coefficient de variation \% & 2,6 & 2,9 \\
\hline
\end{tabular}

\section{CONCLUSION}

La teneur en citrate peut être déterminée par la formation d'un complexe Cu-citrate puis par la mesure de l'absorption à $280 \mathrm{~nm}$. Cette méthode est rapide et les résultats sont obtenus avec une bonne précision. Pour l'application au lait, l'élimination des protéines est nécessaire et peut être réalisée par ultrafiltration ; le dosage est alors effectué sur l'ultrafiltrat qui contient seulement le citrate soluble du lait. Le citrate colloïdal associé à la caséine micellaire, et qui représente environ $10 \%$ du citrate total du lait (Pyne et Mc Gann, 1960), est éliminé pendant l'ultrafiltration. La comparaison des teneurs en citrate obtenues par la méthode du complexe $\mathrm{Cu}$ et par la méthode de White et Davies (1963) prise comme référence (tab. 2) a montré que les valeurs moyennes ne diffèrent pas significativement. Le phosphate soluble du lait pourrait théoriquement interférer avec la détermination du citrate et conduire à une surestimation de $4 \%$ de la teneur réelle en citrate, compte tenu des teneurs respectives en phosphate et en citrate présentes dans l'ultrafiltrat $(0.01$ et $0.008 \mathrm{M} / \mathrm{l}$ ) et des coefficients respectifs d'extinction moléculaire (48 et 1416). En fait, les résultats expérimentaux (tab. 2) montrent que les teneurs de citrate déterminées par la méthode complexe $\mathrm{Cu}$ ne sont pas aussi affectées qu'on pourrait s'y attendre, comme le montre la comparaison avec la méthode de White et Davies (1963).

\section{Bibliographie}

Holt (C.) and MUIR (D. D.) (1979). - Inorganic constituents of milk: I .Correlation of soluble calcium with citrate in bovine milk. Journal of Dairy Research, $58,433-439$. 
MARIER (J. R.) and Boulet (M.) (1958). - Direct determination of citric acid in milk with an improved pyridin-acetic anhydride method. Journal of Dairy Science, 41, 1683-1692.

Marsili (R. T.), Ostapenko (H.) Simmons (R. E.) and Green (D. E.) (1981). - High performance liquid chromatographic determination of organic acids in dairy products. Journal of Food Science, 46, 52-57.

MutzelbuRG (I. D.) (1979). - An enzymatic method for the determination of citrate in milk. The Australian Journal of Dairy Technology, 34, 82-84.

Pyne (G. T.) and Mc GanN (T.C.A.) (1960). - The colloïdal phosphate of milk. II. Influence of citrate. Journal of Dairy Research, 27, 9-17.

White (J. C. D.) and Davies (D. T.) (1963). - The determination of citric acid in milk and milk sera. Journal of Dairy Research, 30, 171-189. 